\title{
Status of the Brueckner-Hartree-Fock approximation to the nuclear matter binding energy with the Paris potential
}

\author{
H.-J. Schulze, J. Cugnon, and A. Lejeune \\ Université de Liège, Institut de Physique B5, Sart Tilman, B-4000 Liège 1, Belgium \\ M. Baldo and U. Lombardo \\ Dipartimento di Fisica, Università di Catania, Corso Italia 57, I-95129 Catania, Italy
}

(Received 12 May 1995)

\begin{abstract}
A new calculation of the binding energy of nuclear matter in the Brueckner-Hartree-Fock approximation with the Paris potential using the standard and continuous choices of single particle energies is presented, paying special attention to the numerical accuracy and higher partial waves. Comparison with other calculations is made and the accuracy of the state of the art for the Brueckner-Hartree-Fock method is assessed.
\end{abstract}

PACS number(s): 21.65.+f, 21.10.Dr, 21.60.Jz

It is generally considered that the Brueckner-Hartree-Fock (BHF) method $[1,2]$ gives a good estimate of the binding energy of infinite nuclear matter, although there is no really precise evaluation of higher order terms in the BetheGoldstone expansion. Moreover, even for a given nucleonnucleon potential, the various results found in the literature may appear scattered. For instance, for the Paris potential [3], which is one of the most microscopically founded potentials, the published results for the BHF method differ, around normal nuclear matter density, by more than a few $\mathrm{MeV}$ [4-11], as pointed out in [11] and [12]. In particular the results of [4] and [6] are in serious disagreement with each other and the rest of the calculations.

Besides the choice between the so-called continuous and gap prescriptions for the intermediate states, these differences basically arise from the numerical methods, e.g., discretization in $r$ space or in $p$ space, number of partial waves, integration grids, and iteration procedure. One has also to consider that sometimes separable versions of the Paris potential $[13,14]$ are used. We believe it is the right time to reanalyze the situation, to improve it by a new detailed calculation, and to assess the accuracy that can be achieved nowadays in the BHF calculations.

Let us quickly recall the definition of the BHF method. The $G$ matrix corresponding to the $N N$ potential $V$ is given by

$$
G(w)=V+\sum_{p, p^{\prime}} V \frac{\left|p p^{\prime}\right\rangle Q\left(p, p^{\prime}\right)\left\langle p p^{\prime}\right|}{w-e(p)-e\left(p^{\prime}\right)} G(w),
$$

with $e(k)=k^{2} / 2 m+U(k)$, and the single particle potential

$$
U(k)=\operatorname{Re} \sum_{k^{\prime}<k_{F}}\left\langle k k^{\prime}\left|G\left[e(k)+e\left(k^{\prime}\right)\right]\right| k k^{\prime}\right\rangle
$$

The binding energy per nucleon is given by

$$
\frac{B}{A}=\sum_{k<k_{F}}\left(\frac{k^{2}}{2 m}+\frac{1}{2} U(k)\right) \text {. }
$$

$0556-2813 / 95 / 52(5) / 2785(4) / \$ 06.00$
These equations (to be solved self-consistently) define the BHF scheme with the continuous choice of the single particle energies. For the gap (or standard) choice, $U(k)$ is set to zero for $k>k_{F}$. The continuous choice is expected to yield a faster convergence of the Bethe-Goldstone expansion [2]. There is an intermediate choice, adopted in Ref. [7], which consists in introducing Eq. (2) up to $k=k_{M}$ (larger than $k_{F}$ ) and in neglecting the potential energy beyond this value. The $G$ matrix can also be obtained from the correlated wave function, which fulfills an integral equation, similar to Eq. (1), but in $r$ space [2]. In all the calculations we discuss here an angle-averaged form of the Pauli operator $Q$ in Eq. (1) is used and can thus be considered as part of the definition of the BHF scheme (see, however, Ref. [15]).

In this present work, we recalculate the binding energy per nucleon of nuclear matter, with the Paris potential and with the standard and the continuous choices, including partial waves beyond $\ell=4$ (listed in Table I). The method is the discretization of the correlated wave functions in $r$ space. We pay particular attention to the following points:

(1) In contradistinction with previous works [2,6], we abandon the effective mass approximation in the calculation of the Green function appearing in Eq. (1), keeping the true spectrum.

(2) We use a much finer grid (compared to [6]) for the integration on the intermediate states. In fact, we use an adaptative subroutine guaranteeing a relative accuracy better than $1 \%$.

(3) We use the calculated single particle spectrum as the input to the next iteration, and no longer a smooth interpolation of it. Contrary to what is sometimes stated [2], we did not observe any instability in the $k_{F}$ range investigated here and the so-called wiggle (enhancement of the effective mass near $k_{F}$ ) is present at every iteration step, converging quite well.

Our present results differ from those of Ref. [6], especially for $k_{F}$ larger than $1.4 \mathrm{fm}^{-1}$. Apparently, this difference comes mainly from point (2) above: The number of mesh points and the mesh itself for the integration on the 
TABLE I. Partial wave contributions to the binding energy per nucleon (MeV). HPW denotes estimates of contributions of higher partial waves.

\begin{tabular}{|c|c|c|c|c|c|c|}
\hline \multirow[b]{2}{*}{ Ref. } & \multicolumn{3}{|c|}{ Standard BHF $\left(k_{F}=1.40\right)$} & \multicolumn{3}{|c|}{ Continuous BHF $\left(k_{F}=1.36\right)$} \\
\hline & [7] & [5] & Present work & [4] & {$[10,11]^{\mathrm{a}}$} & Present work \\
\hline${ }^{1} S_{0}$ & -16.97 & -16.98 & -16.72 & -16.81 & -16.34 & -16.13 \\
\hline${ }^{3} S_{1}$ & -17.99 & -17.98 & -17.98 & -22.44 & -19.90 & -20.74 \\
\hline${ }^{3} D_{1}$ & 1.69 & 1.69 & 1.70 & 1.48 & $\mathrm{~b}$ & 1.48 \\
\hline${ }^{3} P_{0}$ & -3.84 & -3.83 & -3.97 & -3.61 & -3.16 & -3.71 \\
\hline${ }^{3} P_{1}$ & 11.96 & 11.69 & 11.90 & 10.08 & 9.92 & 10.34 \\
\hline${ }^{1} P_{1}$ & 4.87 & 4.88 & 4.99 & 4.26 & 4.15 & 4.41 \\
\hline${ }^{3} P_{2}$ & -8.14 & -8.14 & -8.13 & -7.73 & -7.84 & -7.59 \\
\hline${ }^{3} F_{2}$ & -0.69 & & -0.68 & -0.61 & c & -0.59 \\
\hline${ }^{1} D_{2}$ & -3.21 & -3.21 & -3.22 & -2.85 & -2.74 & -2.86 \\
\hline${ }^{3} \mathrm{D}_{2}$ & -4.65 & -4.65 & -4.67 & -4.21 & -3.98 & -4.23 \\
\hline Sum & $-36.28^{\mathrm{d}}$ & -36.53 & $-36.10^{\mathrm{d}}$ & -42.44 & -39.89 & -39.62 \\
\hline${ }^{3} D_{3}$ & 0.13 & 0.13 & 0.11 & -0.00 & & 0.02 \\
\hline${ }^{3} G_{3}$ & 0.25 & & 0.25 & 0.22 & & 0.21 \\
\hline${ }^{1} F_{3}$ & 0.79 & & 0.95 & & & 0.84 \\
\hline${ }^{3} F_{3}$ & 1.84 & & 1.80 & & & 1.58 \\
\hline${ }^{3} F_{4}$ & -0.51 & & -0.51 & & & -0.44 \\
\hline${ }^{3} \mathrm{H}_{4}$ & -0.11 & & -0.11 & & & -0.09 \\
\hline${ }^{1} G_{4}$ & -0.56 & & -0.53 & & & -0.46 \\
\hline${ }^{3} G_{4}$ & -0.87 & & -0.84 & & & -0.72 \\
\hline${ }^{3} G_{5}$ & 0.09 & & 0.09 & & & 0.07 \\
\hline${ }^{3} I_{5}$ & 0.04 & & 0.04 & & & 0.03 \\
\hline${ }^{1} H_{5}$ & & & 0.23 & & & 0.20 \\
\hline${ }^{3} H_{5}$ & & & 0.40 & & & 0.34 \\
\hline${ }^{1} I_{6}$ & & & -0.13 & & & -0.11 \\
\hline${ }^{3} I_{6}$ & & & -0.18 & & & -0.15 \\
\hline HPW & 0.31 & 0.86 & & & 1.87 & \\
\hline$B / A$ & -11.2 & -11.2 & -10.8 & -19.2 & -15.0 & -15.3 \\
\hline
\end{tabular}

${ }^{\text {a Separable Paris potential. }}$

${ }^{\mathrm{b}}$ Contained in ${ }^{3} S_{1}$.

${ }^{\mathrm{c}}$ Contained in ${ }^{3} \mathrm{P}_{2}$.

${ }^{\mathrm{d}}$ Without ${ }^{3} \mathrm{~F}_{2}$.

intermediate states in the Green function were misleadingly chosen in Ref. [6]. We will thus exclude these old results from our discussion and proceed to the comparison of our new results with those of Refs. [4,5,7-11].

We start, in Table I, with a detailed comparison of the partial wave contributions to the binding energy at nuclear matter density in both the standard and continuous schemes. The standard scheme is numerically much easier to realize and we therefore expect a good agreement between our and two independent, previous calculations $[5,7]$. This is indeed confirmed by comparing the values for the various partial waves for the three calculations, where we find a maximal variation of about $5 \%$. For the sum of the partial waves up to ${ }^{3} \mathrm{D}_{2}$, the variation is even only about $1 \%(0.4 \mathrm{MeV})$.

The situation concerning the continuous choice is worse. There is only one exactly comparable calculation with the full Paris potential [4], in which, however, only partial waves up to $\ell=2$ are consistently taken into account. The total results are not corrected for higher partial waves and there- fore unrealistic. Another comparable calculation [10,11] employs a separable version of the Paris potential [14], ${ }^{1}$ again only up to $\ell=2$. In this case, however, an empirical correction for higher partial waves, derived from the standard choice results of Ref. [7], is applied. Nevertheless, we can again compare the partial waves up to ${ }^{3} D_{2}$, and find here a variation of about $8 \%$ for the three calculations. This is mainly due to the contribution of the ${ }^{3} S_{1}$ channel in Ref. [4], which is fairly large and renders the total sum about $3 \mathrm{MeV}$ larger than for the other two calculations, which agree within $2 \%$ on the $\ell \leqslant 2$ contribution.

With variations of this size and in view of the discrepancy observed with Refs. [5,7] (see further below), it is fairly important to estimate the numerical accuracy of our results. Apart from the number of partial waves that are taken into

\footnotetext{
${ }^{1}$ We do not compare with the results of Refs. [8,9], which use an older version of the separable Paris potential [13].
} 
account, this is influenced by four discretization procedures that are necessary for the numerical treatment: (1) the grid in $r$ space that is used for the numerical diagonalization of the Bethe-Goldstone equation and for the determination of the $G$-matrix elements from the correlated wave functions by numerical integration, (2) the grid in $k$ space for the computation of the intermediate nucleon-nucleon Green functions (see remarks above), (3) the grid for the $k^{\prime}$ integration in Eq. (2), in order to compute the single particle potential from the $G$ matrix [most often (but not in our case) this integration is replaced by an integration over the relative momentum between the nucleons $k$ and $k^{\prime}$, with a suitably averaged total momentum, thus introducing a further source of inaccuracy], and (4) the grid for the discretization of the single particle potential, i.e., the points in $k$ space at which the selfconsistency Eq. (2) is enforced.

Each of these grids is characterized by (i) a typical interval spacing and (ii) a maximum cutoff value. In order to achieve acceptable performance, the choice of these parameters has to be compromised. We can, however, vary them independently, in order to estimate the error of the final results. By doing so, we estimate the accuracy of the calculated values of the single particle potential $U(k)$, including the effects of nonexplicitly treated partial waves $\ell \geqslant 5,{ }^{2}$ to be $\pm 2 \%$. This relatively small value translates into a much larger inaccuracy of the binding energy $B / A$, however, since this is the result of a subtraction of two large numbers: At nuclear matter density $k_{F}=1.36 \mathrm{fm}^{-1}$ we obtain an accuracy of $\pm 0.6 \mathrm{MeV}(4 \%)$, whereas at $k_{F}=1.8 \mathrm{fm}^{-1}$ it is $\pm 1.3 \mathrm{MeV}(11 \%)$. These error estimates are slightly smaller than those of Ref. [5], whereas the other publications do not give errors.

We then compare in Fig. 1 the results of the various calculations for the total binding energy per nucleon, including corrections for higher partial waves. For the standard calculations, we find good agreement of the three curves with a maximum variation of $\approx 1 \mathrm{MeV}$ within the range $k_{F}=1.2-1.8 \mathrm{fm}^{-1}$. For the continuous choice, the only reasonable comparison can be made with Refs. [10,11] (separable Paris potential), since in Ref. [4] higher partial waves are not included. Here the deviation of the two curves lies within $1.2 \mathrm{MeV}$. For the sake of comparison we display in the same figure two curves that are obtained in calculations with the Paris potential closely related to the continuous BHF scheme: (a) the "model space" calculation of Ref. [7], in which the self-consistency condition Eq. (2) is only maintained up to $k_{M}=2 k_{F}$, and (b) the work of Ref. [5], in which the so-called three-body cluster energies $D_{3}^{c}$ are added to the standard BHF values. According to the authors of Ref. [5], this is basically equivalent to the continuous choice.

We observe that compared to the continuous BHF results the binding energy in the model space calculation is systematically smaller at low densities, whereas the three-body cluster calculation gives more binding energy at, and a saturation curve shifted to, larger densities.

Finally, Fig. 1 shows also the result of the continuous

\footnotetext{
${ }^{2} \mathrm{We}$ estimate that this contribution is less than $0.1 \mathrm{MeV}$ at $k_{F}=1.4 \mathrm{fm}^{-1}$.
}

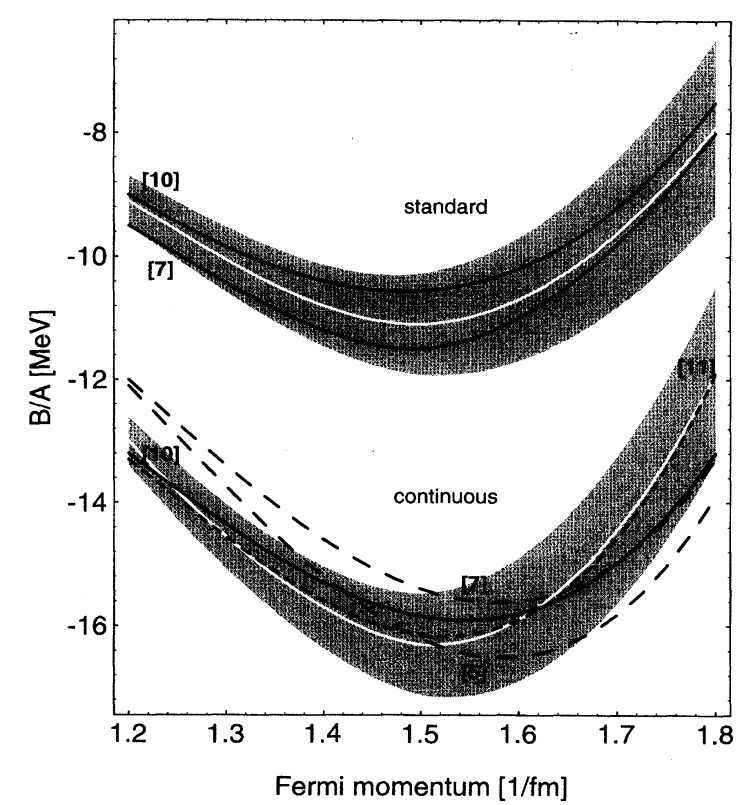

FIG. 1. Saturation curves for various BHF calculations using the standard choice or the continuous choice. Our results are represented by the white curves with the shaded areas indicating the accuracy, and compared with previous results $[7,10]$ (solid curves). We also display the results of alternative choices close to the continuous choice [5,7] (long dashed), and those of a calculation with the Argonne $V_{14}$ potential [11] (short dashed curve).

BHF calculation with the Argonne $V_{14}$ potential of Ref. [11], which turns out to be in amazing agreement with our Paris result.

In summary, we presented the first calculation of the binding energy of symmetric nuclear matter in the BHF approximation with the Paris potential and with the continuous choice of single particle potentials that takes into account all partial waves up to $\ell=4$ and the uncoupled $\ell=5,6$ waves. Our results for the standard choice are in very good agreement with two previous calculations, indicating that our method of solving the Bethe-Brueckner equation in $r$ space is as reliable as the treatment in $p$ space in the other works. For the continuous choice we obtain slightly more binding than the only directly comparable calculation, which, however, uses a separable form of the Paris potential, and only estimates the effects of partial waves $\ell>2$. We assess the accuracy of our calculation to be better than $\pm 2 \%$ for the values of the single particle potentials. For the binding energy at nuclear matter density $k_{F}=1.36 \mathrm{fm}^{-1}$ we find $B / A=15.3 \pm 0.6 \mathrm{MeV}$ and the minimum of the saturation curve lies at $k_{F} \approx 1.51 \mathrm{fm}^{-1}$ and $B / A \approx 16.3 \mathrm{MeV}$. Around these values of $k_{F}$ the dispersion of the binding energy evaluated by the most sophisticated calculations using the Paris (or Argonne) potential is less than $1.2 \mathrm{MeV}$.

This work was supported by EU Contract No. ERBCHRX-CT92-0075. 
[1] K. A. Brueckner and J. L. Gammel, Phys. Rev. 109, 1023 (1958).

[2] J.-P. Jeukenne, A. Lejeune, and C. Mahaux, Phys. Rep. C 25, 83 (1976).

[3] M. Lacombe et al., Phys. Rev. C 21, 861 (1980).

[4] M. A. Matin and M. Dey, Phys. Rev. C 27, 2356 (1983); C 29, 334(E) (1984).

[5] B. D. Day and R. B. Wiringa, Phys. Rev. C 32, 1057 (1985).

[6] A. Lejeune, P. Grangé, M. Martzolff, and J. Cugnon, Nucl. Phys. A453, 189 (1986).

[7] T. T. S. Kuo, Z. Y. Ma, and R. Vinh Mau, Phys. Rev. C 33, 717 (1986).

[8] M. Baldo, I. Bombaci, L. S. Ferreira, G. Giansiracusa, and U. Lombardo, Phys. Lett. B 209, 135 (1988).
[9] M. Baldo, I. Bombaci, G. Giansiracusa, U. Lombardo, C. Mahaux, and R. Sartor, Phys. Rev. C 41, 1748 (1990).

[10] M. Baldo, I. Bombaci, G. Giansiracusa, and U. Lombardo, J. Phys. G 16, L263 (1990).

[11] M. Baldo, I. Bombaci, G. Giansiracusa, and U. Lombardo, Phys. Rev. C 43, 2605 (1991).

[12] C. Mahaux and R. Sartor, Nuclear Matter and Heavy Ion Collisions, edited by M. Soyeur et al., Vol. 205 of NATO Advanced Study Institute Series B: Physics (Plenum Press, New York, 1989).

[13] J. Heidenbauer and W. Plessas, Phys. Rev. C 30, 1822 (1984).

[14] J. Heidenbauer and W. Plessas, Phys. Rev. C 32, 1424 (1985).

[15] T. Cheon and E. F. Redish, Phys. Rev. C 39, 331 (1989). 


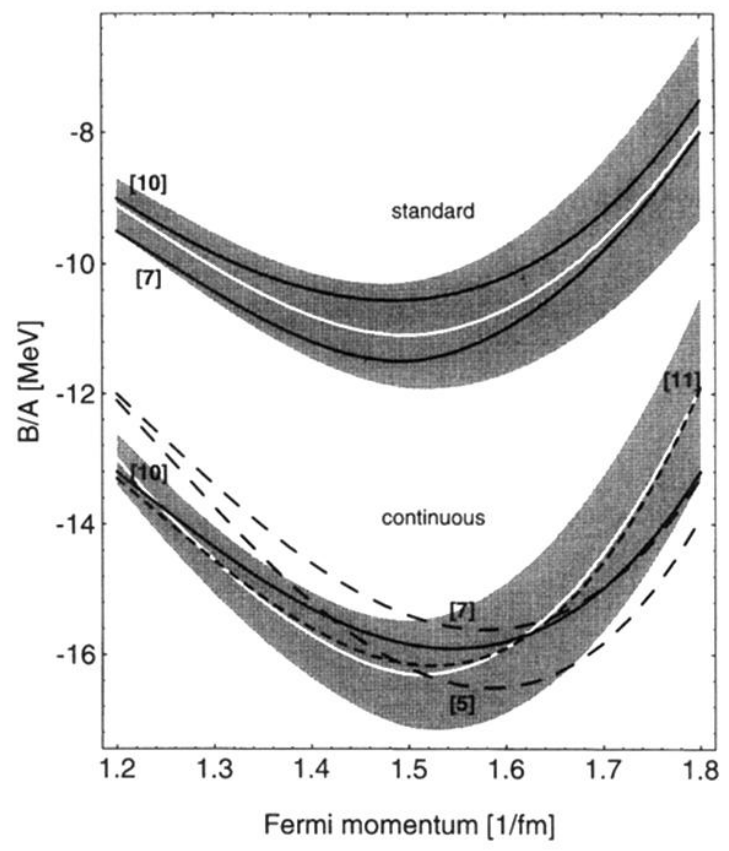

FIG. 1. Saturation curves for various BHF calculations using the standard choice or the continuous choice. Our results are represented by the white curves with the shaded areas indicating the accuracy, and compared with previous results $[7,10]$ (solid curves). We also display the results of alternative choices close to the continuous choice $[5,7]$ (long dashed), and those of a calculation with the Argonne $V_{14}$ potential [11] (short dashed curve). 